\title{
Correction to: Characterization and molecular cloning of novel isoforms of human spermatogenesis associated gene SPATA3
}

\author{
Published online: 7 January 2020 \\ (c) Springer Nature B.V. 2020

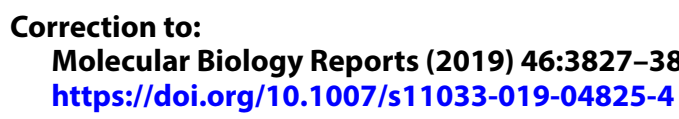

Baixu Zhou ${ }^{1,2} \cdot$ Chunli Wei $^{1} \cdot$ Md. Asaduzzaman Khan ${ }^{1} \cdot$ Hanchun Chen ${ }^{3} \cdot$ Junjiang Fu ${ }^{1}[$

Unfortunately, as for the second institute name of first author Baixu Zhou, "Department of Gynecology and Obstetrics, Guangzhou Women and Children's Hospital, Guangzhou, Guangdong, China", should be "Department of Gynecology

and Obstetrics, Guangdong Women and Children Hospital, Guangzhou, Guangdong, China".

Publisher's Note Springer Nature remains neutral with regard to jurisdictional claims in published maps and institutional affiliations.

The original article can be found online at https://doi.org/10.1007/ s11033-019-04825-4.

Junjiang Fu

fujunjiang@hotmail.com; fujunjiang@swmu.edu.cn

1 Key Laboratory of Epigenetics and Oncology, the Research Center for Preclinical Medicine, Southwest Medical University, 3-319, Zhongshan Rd, Luzhou 646000, Sichuan, China

2 Department of Gynecology and Obstetrics, Guangdong Women and Children Hospital, Guangzhou, Guangdong, China

3 Department of Biochemistry and Molecular Biology, School of Life Sciences, Central South University, Changsha 410013, Hunan, China 